\title{
REVOLUTA regulates meristem initiation at lateral positions
}

\author{
Denichiro Otsuga ${ }^{1}$, Bernadette DeGuzman ${ }^{2, \dagger}$, Michael J. Prigge ${ }^{2}$, Gary N. Drews ${ }^{1}$ and Steven E. Clark ${ }^{2, *}$ \\ ${ }^{1}$ Department of Biology, University of Utah, Salt Lake City, UT 84112-0840, USA, and \\ ${ }^{2}$ Department of Biology, University of Michigan, Ann Arbor, MI 48109-1048, USA
}

Received 21 August 2000; revised 7 November 2000; accepted 20 November 2000.

*For correspondence (fax +1 734647 0884; e-mail clarks@umich.edu).

${ }^{\dagger}$ The first two authors contributed equally to this work.

\begin{abstract}
Summary
While the shoot apical meristem (SAM) is indirectly responsible for the initiation of all above-ground postembryonic organs, in most plants the vast majority of these organs are directly initiated by lateral meristems. In Arabidopsis thaliana, the lateral meristems include flower meristems (FMs), which form on the flanks of the SAM, and lateral shoot meristems (LSMs), which develop in leaf axils. While significant progress has been made on the molecular genetic basis of SAM initiation during embryo development, relatively little is known about the initiation of meristems at lateral positions. Here we have characterized the phenotypic consequences and genetic interactions of mutations in the REVOLUTA (REV gene, with an emphasis on the role of REV in lateral meristem initiation. Our observations indicate that $R E V$ is required for initiation of both LSMs and FMs, and likely acts in the same pathway as, and upstream of, known meristem regulators. We identified the REV gene and found it encodes a predicted homeodomain/leucine zipper transcription factor that also contains a START sterol-lipid binding domain. $R E V$ is the same as the IFL gene. REV was expressed at the earliest stages of LSM and FM formation. Within the inflorescence shoot meristem, REV expression appeared to predict 3-5 incipient flower primordia on the flanks of the SAM, and REV expression at stage 1 and stage 2 matched that of WUS and STM, respectively. We propose that REV acts at lateral positions to activate the expression of known meristem regulators.
\end{abstract}

Keywords: meristem, axillary, homeodomain, flower.

Introduction

In Arabidopsis and most other angiosperms, the shoot apical meristem (SAM) is the ultimate source of all aboveground postembryonic organs (e.g. stems, leaves and flowers). However, the majority of these organs are not initiated directly by the SAM, but are instead initiated by lateral meristems. In Arabidopsis, the lateral meristems include flower meristems (FM), which form on the flanks of the SAM, and lateral shoot meristems (LSM), which develop in leaf axils. Each Arabidopsis FM initiates 16 additional organs and each LSM gives rise to a side branch containing leaves and flowers (Smyth etal., 1990). The overall morphology and reproductive capability of a plant is determined in part by the number, position and growth of lateral meristems.

In recent years, much has been learned about SAM initiation and maintenance (Eshed and Bowman, 2000).
The SAM initiation occurs during embryogenesis, and is a complex process requiring the action of several welldefined genes including STM (SHOOT MERISTEMLESS), WUS (WUSCHEL), ZLL/PND (ZWILLE/PINHEAD), CUC1 (CUP SHAPED COTYLEDON) and CUC2 (Aida et al., 1997; Barton and Poethig, 1993; Endrizzi etal., 1996; Jürgens et al., 1994; Laux et al., 1996; Long and Barton, 1998; Lynn et al., 1999; Mayer et al., 1998; Moussian et al., 1998). These genes act over a relatively long developmental period, starting with the 16-cell embryo. Post-embryonically, the SAM maintains a nearly constant number of undifferentiated stem cells, and directs flanking progeny cells toward organ formation and eventual differentiation (Clark, 1997; Steeves and Sussex, 1989). Genes important for SAM maintenance include CLAVATA1 (CLV1), CLV2, CLV3, STM and WUS. STM and WUS are required to maintain the 
Table 1. Rev alleles discussed in this paper

\begin{tabular}{lllllll}
\hline Allele & Isolate & Mutagen & Background & Lesion $^{\text {a }}$ & Predicted effect ${ }^{\text {b }}$ & Origin \\
\hline$r e v-1$ & - & EMS & No-O & C & splice acceptor loss & Talbert et al. (1995) \\
$r e v-3$ & - & EMS & Col & C1823 to T & T608 to I & Talbert et al. (1995) \\
$r e v-5$ & $s p z-1$ & EMS & Col & C779 to T & A260 to V & Alvarez (1994) \\
$r e v-6$ & $v a m-1$ & EMS & clv3-1 & C1036 to T & R346 to stop & Pogany etal. (1998) \\
$r e v-7$ & $v a m-2$ & TDNA & RLD & nd & nd & Pogany etal. (1998) \\
$r e v-8$ & tj72 & TDNA & Ler & intron 1 insertion & RNA instability & Chen et al. (1999) \\
\hline
\end{tabular}

${ }^{a}$ Change in DNA sequence relative to wild-type ecotype, numbered from the start ATG. ${ }^{b}$ Change in mRNA structure or predicted protein numbered from the start methionine. ${ }^{\mathrm{c}}$ See Ratcliffe et al. (2000) for description of rev-1 DNA sequence. nd, not determined.

population of undifferentiated cells (Barton and Poethig, 1993; Laux etal., 1996), and the CLV loci are required to regulate the balance of these cells between proliferation and differentiation (Clark etal., 1993; Clark etal., 1995; Kayes and Clark, 1998; Laufs et al., 1998). All of these genes are expressed in specific regions of the SAM: WUS is expressed in a small number of centrally located cells in the corpus of the SAM, CLV1 and CLV3 are expressed in a central region of the SAM in the corpus and tunica, respectively, and STM is expressed in a central region of the shoot meristem in all cell layers (Clark etal., 1997; Fletcher etal., 1999; Long and Barton, 2000; Long etal., 1996; Mayer et al., 1998).

In contrast to the SAM, little is known about lateral meristem initiation. The morphology of FM and LSM formation has been described in Arabidopsis (Grbic and Bleecker, 2000; Smyth et al., 1990) and many other species (Steeves and Sussex, 1989), and several mutations affecting FM and LSM formation have been reported. These mutants include the tomato lateral suppressor (/s) mutant (Schumacher etal., 1999) and the Arabidopsis revoluta (rev; Talbert et al., 1995), zwille/pinhead (zll; McConnell and Barton, 1995; Moussian et al., 1998), caf (Jacobsen etal., 1999), and argonaute1 (ago1) (Bohmert etal., 1997) mutants. Of these, the $/ s$ and rev mutations specifically affect lateral meristem formation (i.e. these mutations affect both LSM and FM formation). zll and ago 1 mutations affect the primary SAM in addition to the LSMs. An additional class of mutants, including the recently characterized axr1 mutant (Stirnberg et al., 1999), initiate LSMs that arrest early in development. Despite the presence of these mutants, and the fact that many of the corresponding genes have been cloned, little is known about the molecular mechanisms of LSM and FM initiation.

Many of the genes expressed in the SAM are also expressed in the lateral meristems. For example, WUS, STM, CLV1 and CLV3, while not expressed in very young FMs (early stage 1), become re-expressed during early flower development (stages 1 and 2). These data suggest that many of the genes required for SAM formation may also be required for lateral meristem formation.
Furthermore, these data suggest that very young lateral meristems do not retain meristem identity as they are separated from the apex, but instead have meristem identity reactivated shortly after their initiation. This raises the question of how the SAM-expressed genes become reactivated in lateral meristems.

To understand the mechanisms of lateral meristem initiation, we carried out an analysis of the rev mutant. The original description of REV detailed pleiotropic effects of loss-of-function mutations in this gene (Talbert etal., 1995). These defects included a reduction in LSM and FM initiation, as well as leaf and flower organ defects. Rev alleles were subsequently described as variable meristem (vam), a modifier of the clv mutant phenotype (Pogany etal., 1998). Based on our prior analysis of rev/vam mutants, we hypothesized that REV was required to reactivate meristem activity in lateral shoot and flower meristem positions. To test this we have characterized the meristem defects of rev mutants and the genetic interactions with other meristem regulators. We present findings that indicate that $R E V$ is required for lateral meristem initiation, and that REV acts upstream of the $C L V$ loci, as well as in the same pathway as STM in lateral meristem initiation. We isolated the $R E V$ gene and show that it encodes a group III homeodomain-zip (leucinezipper) protein. This gene was previously isolated as IFL 1, which is required for proper lignification and differentiation of cortical cells in the stem (Zhong and Ye, 1999). Our results are consistent with the proposed role of $R E V$ in activating meristem activity as lateral positions.

\section{Results}

\section{Isolation of rev mutants}

We isolated three new rev alleles that have been named rev-6, rev-7 and rev-8 (Table 1). Rev-6 was isolated as a modifier of the Clv3-1- $1^{-}$phenotype. Rev-7 was identified in the RLD background. Rev- 6 and rev-7 were previously named vam mutations (Pogany etal., 1998). Rev-8 was previously described as an enhancer of filamentous flower 
Figure 1. Rev mutants exhibit lateral meristem defects.

The majority of cauline leaf axils of rev- 6 mutant plants were bare (a, arrows). Rarely, a filamentous structure (b, arrow) or leaf (c, arrow) was observed. In contrast to wildtype flowers (d), rev mutant flowers sometimes lacked stamens and carpels $(e, f)$. A sepal and petal were removed from the flower in panel (e).

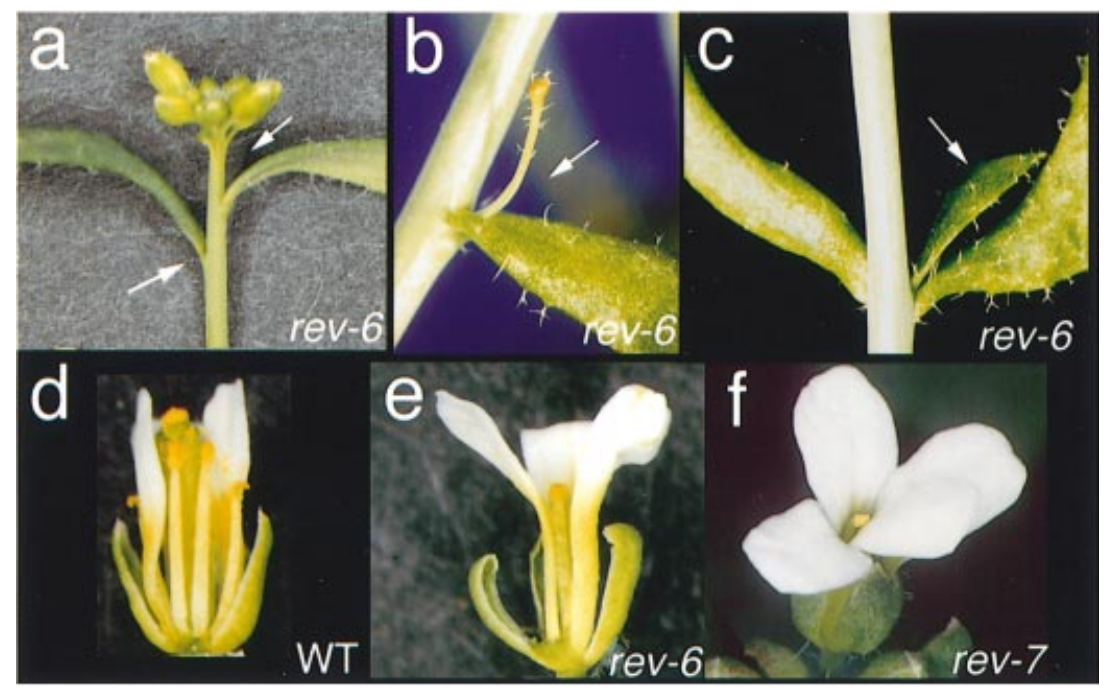

Figure 5. Rev stm and rev wus double mutants.

Rev- 6 enhances stm- 2 vegetative but not flower phenotypes. During vegetative growth, rev-6 stm-2 plants (d,e) developed fewer lateral meristems than stm-2 plants $(a, b)$, and often failed to initiate lateral meristems in axils of leaves (e, arrow). Rev6 stm-2 flowers (f) initiated the same complement of organs as stm-2 flowers (c). While wus- 1 plants continually generated adventitious meristems, which form multiple leaves (g, leaves), rev-6 wus-1 double mutants usually initiated either no organs (i) or a single filamentous structure (h). Samples shown were collected at 25 days after germination. cot, cotyledon. Scale bars are indicated.
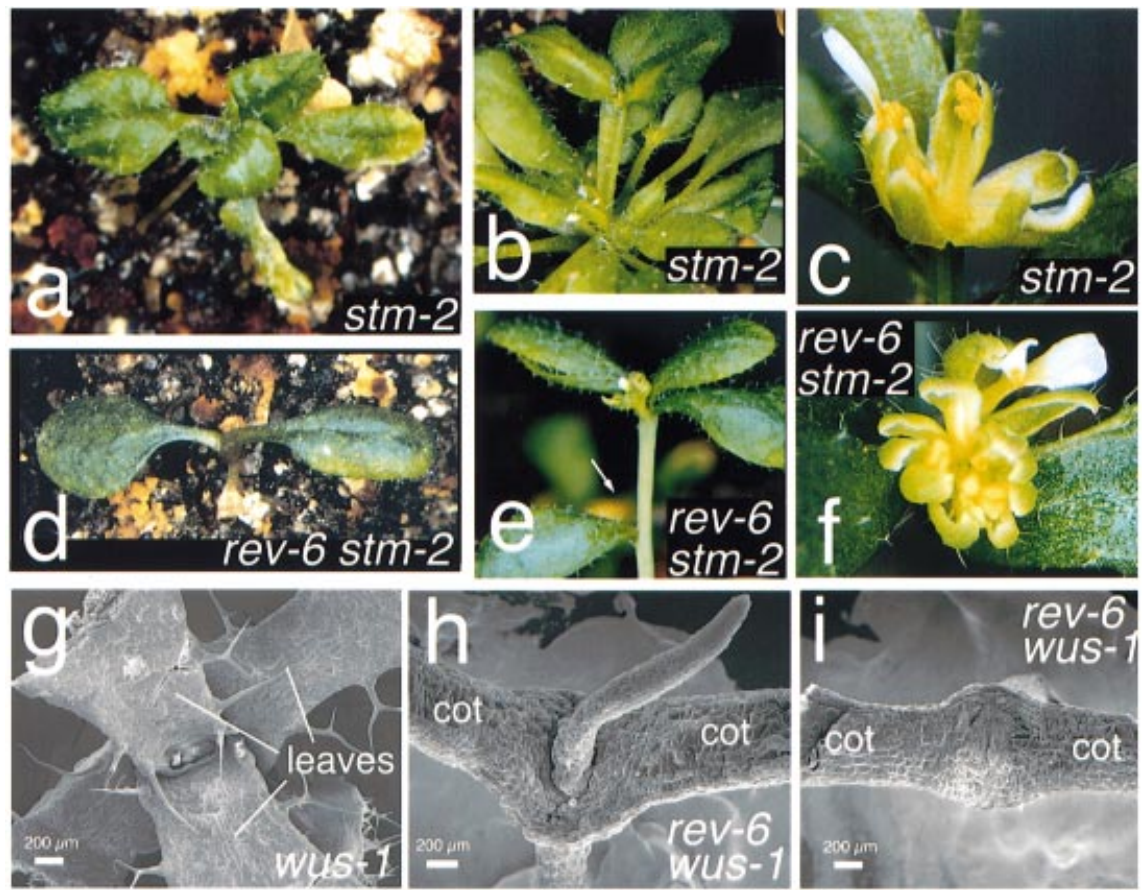

(fil) (Chen etal., 1999). Rev-6 was chosen for further analysis and appears to encode a null allele (see below). Rev- 6 was backcrossed to Landsberg erecta (Ler) three times before phenotypic and genetic characterization.

\section{The rev- 6 mutation affects lateral meristem formation}

Rev- 6 mutants exhibit reduced lateral meristem formation. Most dramatically affected were the LSMs (axillary meristems) of rosette and cauline leaves (Figure 1). In rev-6 mutant plants, approximately $70 \%$ of cauline leaves lacked a lateral meristem (Table 2), and $16 \%$ failed to produce meristems in the axils of any rosette leaves. Decapitation of the primary shoot meristem (SAM) failed to stimulate axillary meristem formation (data not shown). In the place of LSMs, the axils of affected leaves were usually bare, but occasionally developed a differentiated structure that was a leaf or a filamentous structure (Figure 1b,c).

Rev- 6 mutant plants also exhibited a reduction in flower meristem activity. While the majority of the flowers developed normally, $12 \%$ of the flowers initiated only a limited set of flower organs and appeared to have a defective flower meristem (Figure 1e,f). The inner organs were preferentially absent from rev- 6 flowers with defective meristems (Table 3). This pattern of reduction in flower organ number is similar to that seen in wus and fil mutants 
(Chen et al., 1999; Laux et al., 1996). In affected flowers, the floral meristem was reduced in size or nearly absent by stage 3 of flower development (Figure 2d). Other flowers on rev-6 plants were identical to wild-type. Although lateral meristems were dramatically affected in rev- 6 mutants, SAM size and the phyllotaxy of flanking primordia appeared normal (Figure 2c).

\section{The rev- 6 phenotypes are affected by polygenic modifiers}

The original rev-1 allele, which was isolated from the Nossen ecotype, exhibited pleiotropic defects in FM activity, LSM activity, leaf morphology (size, shape, color and senescence), and floral organ morphology (Talbert etal., 1995). In contrast, the rev-6 allele in the Ler ecotype showed none of the non-meristematic defects. These dramatic differences in allele severity could either be due to differences between the effect of the mutations on gene function or to modifiers in the different ecotypes. To test this, we crossed rev- 6 in the Ler ecotype with the Columbia ecotype, and scored the phenotypes segregating in the F2 generation. A range of $\mathrm{Rev}^{-}$phenotypes were observed, including those described for the original rev-1 allele. Statistical analysis indicated a correlation between the

Table 2. Rev- 6 reduces frequency of meristem initiation in cauline leaf axils

\begin{tabular}{lllllr}
\hline & \multicolumn{3}{l}{ Frequency of cauline leaves with } & \\
\cline { 2 - 4 } Genotype & Bare axils & Leaf & Filament & Meristem & $n$ \\
\hline rev-6 & 0.64 & 0.05 & 0.02 & 0.29 & 342 \\
rev-6 clv1-4 & 0.76 & 0.05 & 0 & 0.20 & 66 \\
rev-6 clv2-1 & 0.59 & 0.07 & 0 & 0.35 & 121 \\
rev-6 clv3-2 & 0.72 & 0.08 & 0 & 0.16 & 85 \\
\hline
\end{tabular}

All cauline leaves on the primary inflorescence stems of the indicated genotype were assessed for development within their axils of a meristem, filamentous structure (filament), leaf, or no development (bare axils). $n$ indicates the number of cauline leaves analysed. percentage of absent LSMs and the severity of leaf morphology and color. The severity of leaf morphology and color was also correlated with the percentage of meristem-defective flowers; however, the percentage of absent LSMs was independent of the percentage of meristem-defective flowers (see Supplementary Material text at end of paper). These data suggest that the Columbia ecotype contains multiple modifiers of the $\mathrm{Rev}^{-}$phenotype.

$\mathrm{REV}$ is required for ectopic $L S M$ formation in stm, wus and ap1 mutants

Several mutants such as stm, wus and apetala1 (ap1) produce ectopic meristems. Within stm and wus mutants, ectopic meristems are produced in the axils of cotyledons, and within ap1 mutants, ectopic flower meristems are produced in the axils of whorl 1 leaf-like organs. To test whether REV is required for formation of these ectopic meristems, we analysed rev stm, rev wus and rev ap1 doubly mutant strains.

Mutations in the STM gene result in a failure to initiate an embryonic SAM (Barton and Poethig, 1993). Thus, postembryonic growth in these mutants is entirely dependent on ectopic organogenesis. In the weak stm-2 allele, ectopic shoot meristems are formed in the axils of the cotyledons (Clark etal., 1996). Because STM is also required to maintain the undifferentiated cells of shoot and flower meristems, the ectopic meristems in stm-2 plants terminate after initiating a limited number of organs. Further growth is dependent on additional LSMs that are initiated in the axils of existing leaves. To determine whether the ectopic lateral meristems in stm-2 plants required $R E V$ activity, we analysed rev- 6 stm- 2 double mutants. In contrast to stm-2 plants, in which all plants produced ectopic lateral meristems and the majority did so by 10 days after germination, $13 \%$ of rev- 6 stm- 2 plants never formed shoot meristems, and those that did were delayed in meristem initiation (Figure 4). Furthermore, for those rev-6 stm-2 plants that did undergo postembryonic development, there was a dramatic reduction in the

Table 3. Rev-6 is epistatic to $c / v$ mutations in meristem defective flowers

\begin{tabular}{lllllll}
\hline & \multicolumn{2}{l}{ Organ number per flower } & & & \\
\cline { 2 - 6 } Genotype & Sepals & Petals & Stamens & Carpels & $\mathrm{n}$ & $\%$ of total \\
\hline rev-6 & $2.7 \pm 0.13$ & $1.9 \pm 0.25$ & $0.3 \pm 0.11$ & 0 & 42 & $12 \%$ \\
rev-6 clv1-4 & $2.3 \pm 0.11$ & $1.5 \pm 0.26$ & $0.2 \pm 0.07$ & 0 & 33 & $41 \%$ \\
rev-6 clv3-2 & $2.6 \pm 0.09$ & $1.7 \pm 0.16$ & $0.1 \pm 0.05$ & 0 & 69 & $24 \%$ \\
\hline
\end{tabular}

${ }^{a}$ Mean plus standard error for $n$ flowers. Only flowers lacking normal meristematic activity were used for these means (all others are presented in Figure 3). ${ }^{b}$ Percentage of all flowers counted that lacked normal meristematic activity. $n$ indicates the number of flowers analysed. 
number of LSMs and FMs generated (Figure 5). These data suggest that $R E V$ is required for postembryonic development of shoot meristems in stm-2 plants.

The strong stm-1 allele is phenotypically different from the stm-2 allele in that stm-1 plants never form morphologically identifiable shoot meristems (Barton and Poethig, 1993). In stm-1 plants, leaves are occasionally initiated in the axils of the cotyledons. Later leaves are initiated in the axils of existing leaves (Clark etal., 1996). The origin of postembryonic stm-1 leaves is unclear. It has been hypothesized that stm-1 LSMs become entirely committed to the formation of a differentiated leaf (Clark et al., 1996). An alternative possibility is that these leaves are initiated in a meristem-independent manner. We attempted to distinguish between these possibilities by determining if $R E V$ was required for the formation of the postembryonic stm-1 leaves. Rev- 6 stm- 1 plants were generated and the frequency and timing of the postembryonic initiation of leaves was measured and compared to stm-1 single mutants (Figure 4). Rev-6 stm-1 plants exhibited both a delay and a reduction in the total frequency of plants capable of postembryonic organ formation compared to stm-1 plants, indicating a role for $R E V$ in this process.

Mutations in the WUS gene result in phenotypes similar to partial-loss-of-function mutations in the STM gene (e.g. stm-2), namely, the failure to initiate a shoot meristem in the embryo, but the ability to form adventitious shoots postembryonically (Laux et al., 1996). To determine if the adventitious shoot formation in wus plants required $R E V$ activity, rev-6 wus-1 double mutant plants were compared to wus-1 plants for the ability to initiate postembryonic organs. As shown in Figure 4, all wus-1 plants initiated a visible adventitious shoot by 17 days after germination. By contrast, over $50 \%$ of rev- 6 wus- 1 plants never initiated postembryonic organs (Figures 4 and 5i). Among the rev- 6 wus- 1 plants that did initiate organs, most only formed a single filamentous structure between the cotyledons (Figure 5h).

Ap1 flowers develop leaf-like sepals in whorl 1 that often develop flower meristems in their axils (Bowman etal., 1993). To determine if $R E V$ was required for the ectopic flower meristems in ap1 mutants, rev-6 ap1-1 double mutant plants were generated and the percentage of flowers containing axillary flowers was compared with ap1 mutant plants. $36.2 \%$ of ap 1-1 flowers $(n=958)$ produced ectopic flower meristems. By contrast, only $2.8 \%$ of rev-6 ap 1-1 flowers $(n=782)$ produced ectopic flower meristems, which represents a reduction of over $90 \%$. These data indicate that $R E V$ activity is required for the formation of FMs in the axils of whorl 1 bracts in ap 1 flowers.

Ap1 cauliflower (cal) double mutant plants exhibit even more dramatic ectopic lateral meristem formation. The shoot meristem of these plants initiates lateral meristems, that each in turn initiate lateral meristems, in a reiterative process that leads to the formation of hundreds of meristems (Bowman etal., 1993; see Supplementary Material at end of paper, Figure S1). The rev-6 ap 1-1 cal-1 triple mutant plants exhibited a range in reduction of lateral meristem formation, likely
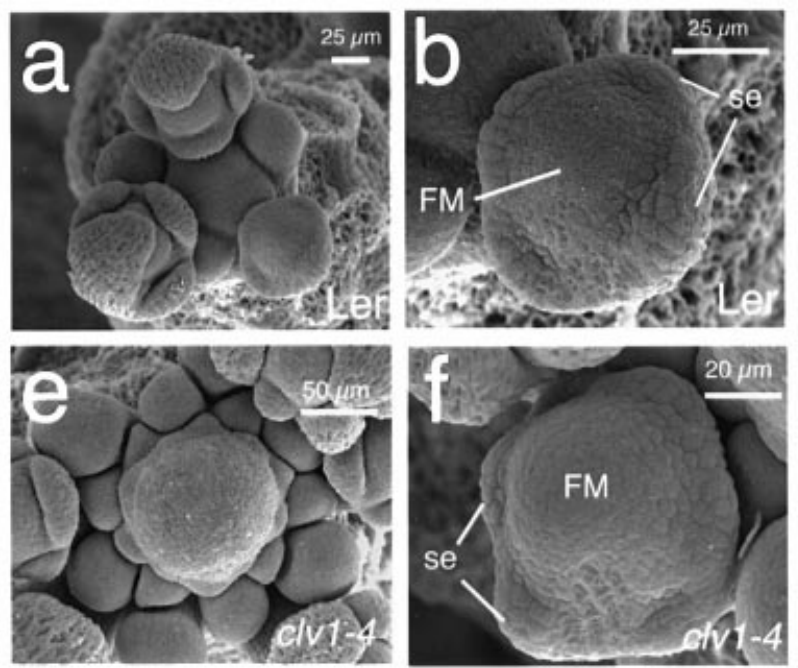
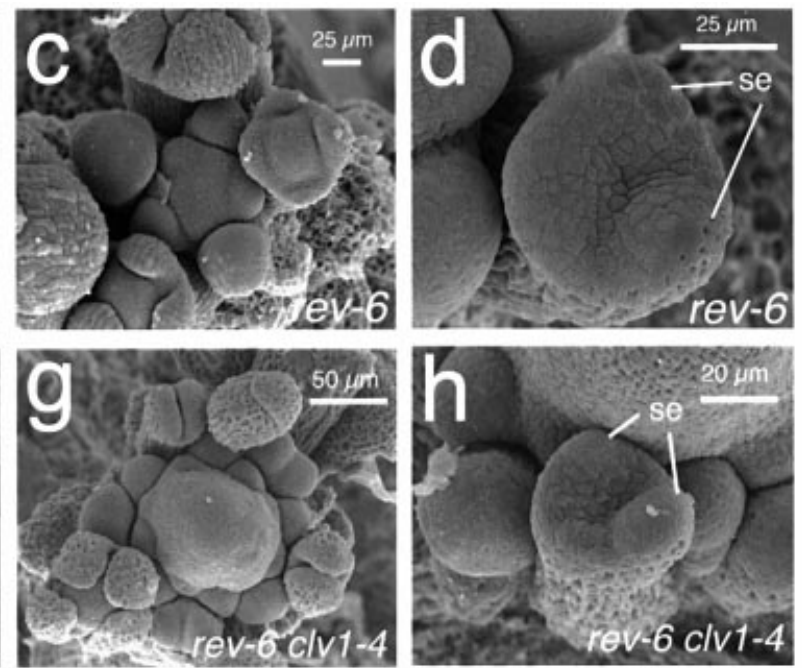

Figure 2. Rev is epistatic to $c / v 1$ and $c / v 3$ in reduced flowers.

Sixteen-day-old wild-type Ler (a,b), rev-6 (c,d), clv1-4 (e,f), and rev-6 clv1-4 (g,h) plants were collected and analysed by scanning electron microscopy (SEM). While rev shoot apical meristems were indistinguishable from wild-type (a,c), stage 3 rev flowers occasionally lacked flower meristems (FM) interior to the sepals (se) (all stages according to Smyth et al., 1990). While rev-6 had no effect on the shoot apical meristems of clv1 mutants (compare e to $\mathrm{g}$ ), reduced stage 3 flowers of rev- $6 \mathrm{clv} 1$ plants (h) lacked the enlarged flower meristems of $c / v 1$ stage 3 flowers (f) and were indistinguishable from rev6 reduced flowers $(d)$. Scale bars are indicated. 


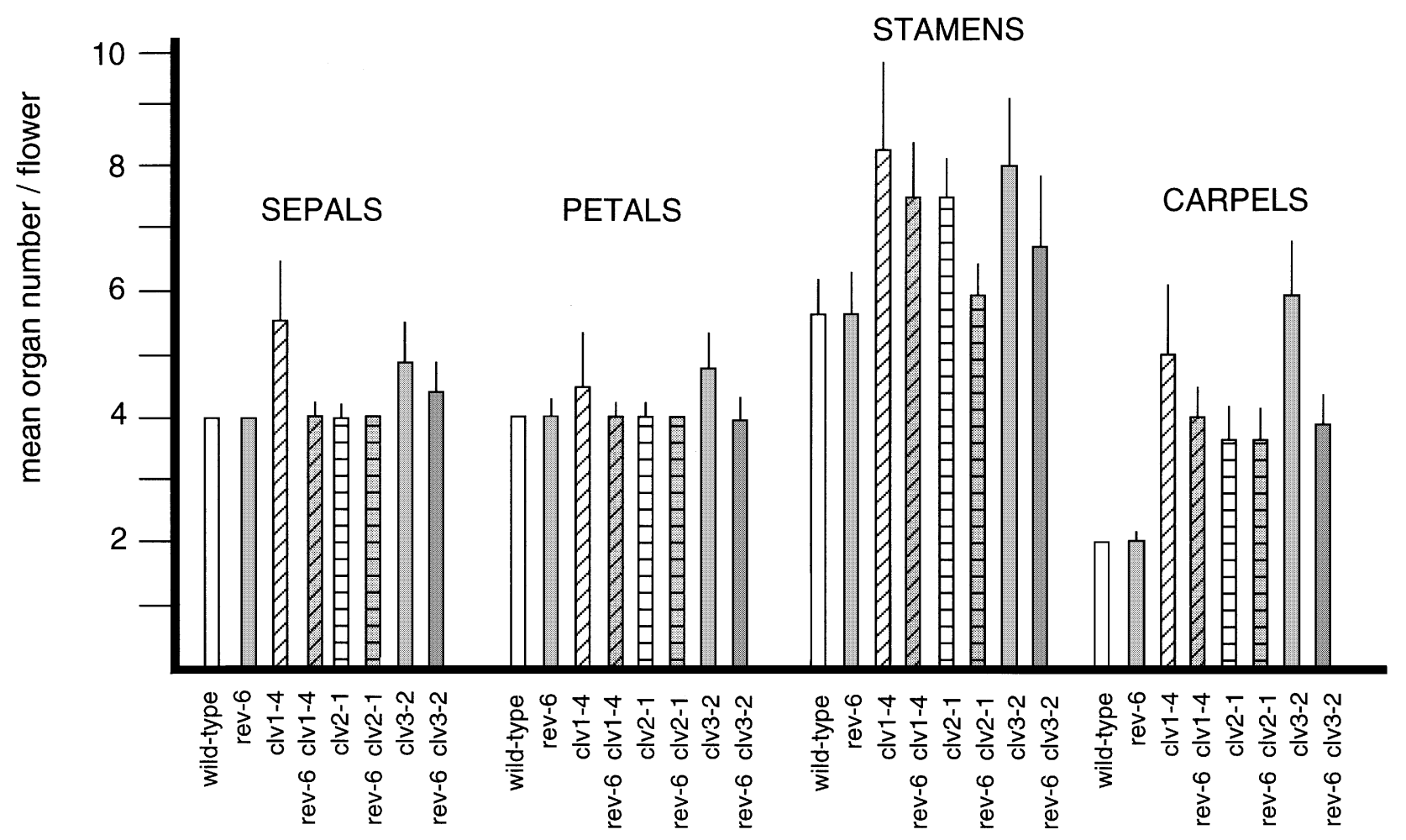

Figure 3. Rev-6 partially suppresses clv mutant flower phenotypes.

The number of flower organs in wild-type Ler, rev-6, clv1-4, rev-6 clv1-4, clv2-1, rev-6 clv2-1, clv3-2, and rev-6 clv3-2 plants were counted. Only complete flowers were included in the calculations of the mean and standard deviation for each genotype (see text). At least 100 flowers were counted for each mean, and only the first 10 flowers on any given plant were counted.

due to the mixed Landsberg/Wassilewskija ecotype of the ap1-1 cal-1 double mutant. At the extreme end of the phenotypic range, no lateral meristems were formed on the flanks of the triple mutant plants (see Supplementary Material at end of paper, Figure S1). The only lateral organs produced were small bract-like or filamentous structures. Other triple mutant plants exhibited a strong reduction in lateral meristem formation, but still initiated a number of lateral shoots, that each initiated small bract-like structures (see Supplementary Material at end of paper, Figure S1). Taken together, these data suggest that $R E V$ is required for the initiation of all postembryonic shoot and flower meristems.

\section{CLV and STM activity appear reduced in rev mutants}

Rev-6 plants exhibited a variably expressive reduced meristem activity at lateral positions. The most severe examples of this were the reduced flowers (flowers with no stamens or carpels) and bare leaf axils, both of which appeared to completely lack meristem activity. If this is indeed the case, the reduced flowers and bare axils should be unaffected by mutations in genes that modulate meristem activity, such as clv, stm and wus mutations. Furthermore, the rev- 6 mutation should attenuate the phenotype of mutants with increased meristem activity such as clv mutants. To test this, we analysed rev- 6 clv and rev- 6 stm doubly mutant strains. As discussed above, rev- 6 wus double mutants failed to undergo postembryonic growth, preventing us from assessing the effect of rev- 6 mutation on wus flower development.

We generated double mutant plants carrying rev- 6 with the clv1-4, clv2-1 or clv3-2 mutations. In these double mutants, the frequency of cauline leaves with bare axils (Table 2) and the phenotype of reduced flowers (Table 3) was similar to that in rev single mutants. A higher percentage of reduced flowers was found in the double mutant plants in comparison to rev- 6 single mutant plants (Table 3). These flowers exhibited clear reductions in floral primordia size at stage 3 (Figure $2 \mathrm{~h}$, data not shown), and initiated a nearly identical number of floral organs compared to the reduced flowers of rev- 6 single mutants (Table 3). The 'complete' flowers (flowers with all organ types) exhibited a $\mathrm{Clv}^{-}$phenotype, but the severity was reduced in comparison to the respective $c / v$ single mutant. 


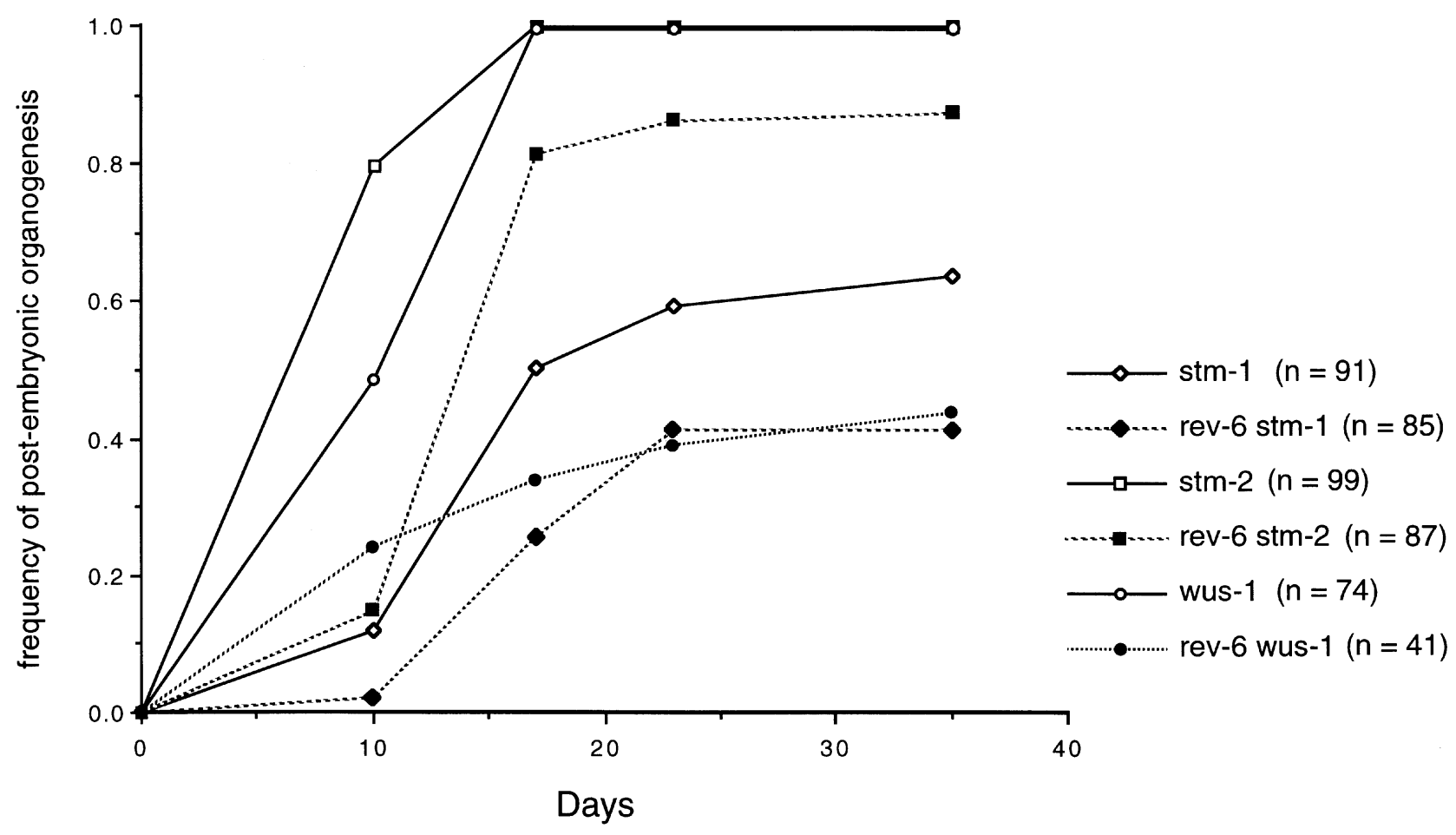

Figure 4. Rev-6 limits postembryonic development of stm and wus mutants.

The ability of stm-1, rev-6 stm-1, stm-2, rev-6 stm-2, wus-1 and rev-6 wus-2 plants to initiate postembryonic organs was measured in tissue culture as described in Experimental procedures. Shown are the proportion of plants for each genotype that had developed postembryonic organs. The numbers of plants monitored for each genotype are indicated $(n)$.

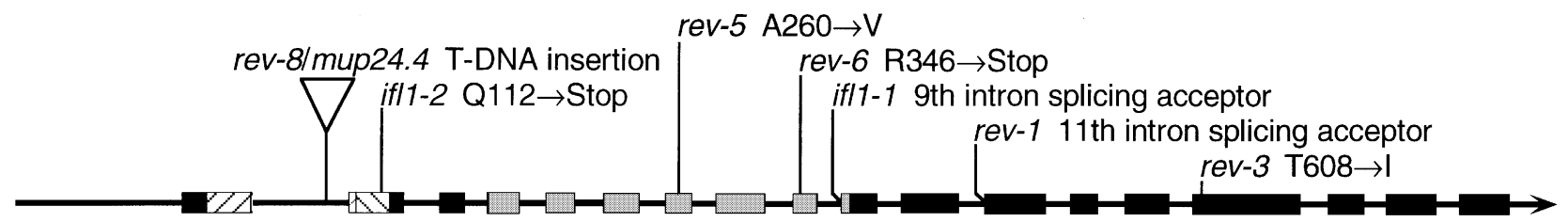

U/AN

HD ZIP START domain

Figure 6. REV encodes a putative transcription factor.

The genomic and mRNA structures for the REVOLUTA gene are shown. Domains encoding the predicted homeodomain (HD), leucine zipper (ZIP) and START domain (START) are indicated. The locations of lesions in rev alleles are shown.

This was especially true in the rev-6 double mutant plants with the strong clv1-4 and clv3-2 alleles (Figure 3). Taken together, these data suggest that the REV gene is required to promote meristem formation and/or activity at lateral positions.

We generated double mutant plants carrying rev- 6 with the weak stm-2 mutation. Stm-2 mutants form shoot meristems that give rise to floral meristems. Stm-2 flowers exhibit limited meristem activity and produce a reduced number of flower organs, especially in the inner whorls of stamens and carpels (Clark etal., 1996). In rev-6 stm-2 double mutant plants, there was a dramatic reduction in the number of floral meristems generated compared to stm-2 single mutants. Those flowers that were initiated contained sepals, petals and stamens (Figure 5f). Thus, the flower meristem phenotype of rev- $6 \mathrm{stm}-2$ was not additive, suggesting a common pathway for REV and STM in floral meristem development. 

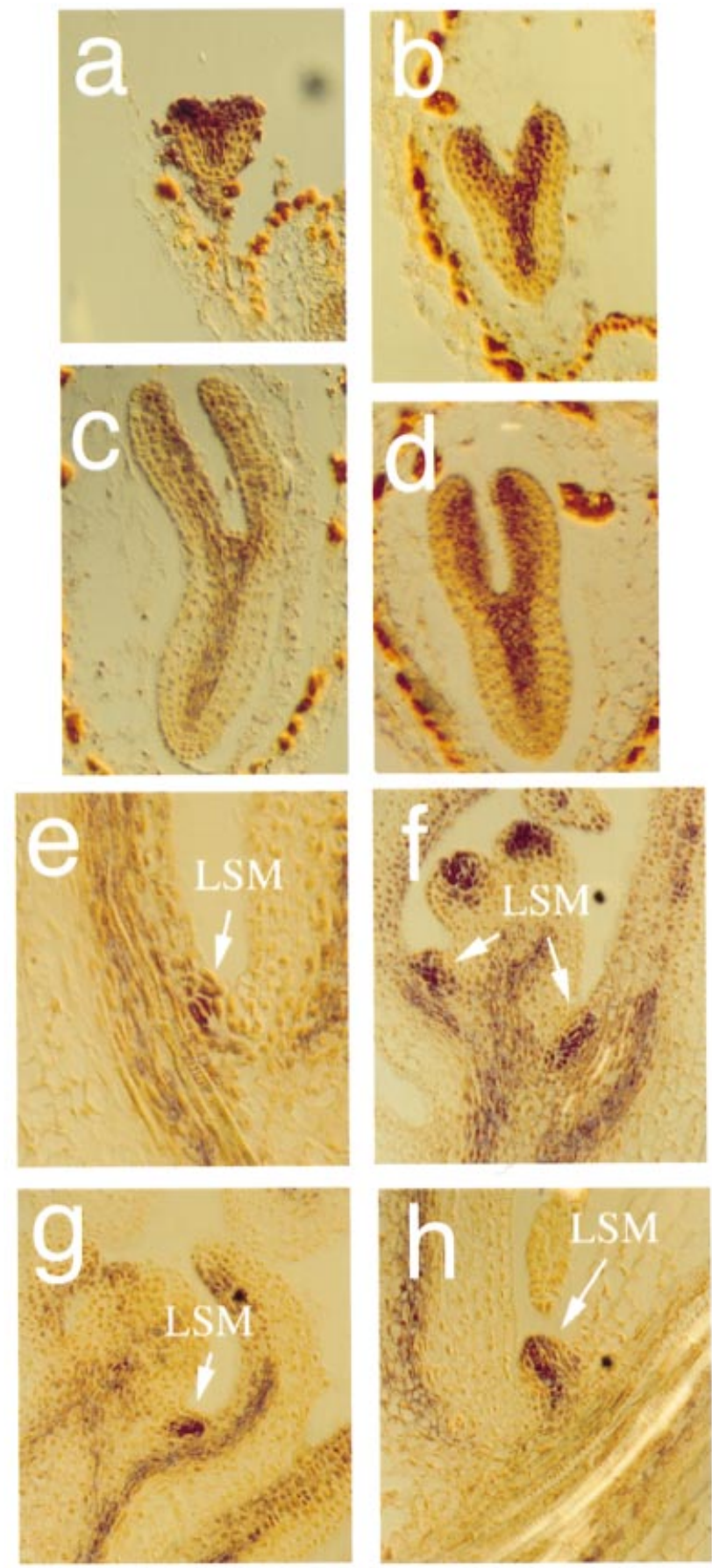

Figure 7. REV expression during embryonic and lateral shoot meristem development.

RNA in situ hybridizations with a REV antisense probe were performed on wild-type embryos at early heart (a), late heart (b), torpedo (d) and walking stick (c) stages. During these stages, REV was expressed throughout the shoot apical meristem, in the adaxial region of the cotyledons, and in the vascular precursors of the hypocotyl and root. REV expression in the earliest stages of lateral shoot meristem (LSM) formation in cauline leaf axils was analysed (e-h). REV was expressed at the earliest sign of LSM proliferation in the leaf axils (e-g), and expanded as the developing meristem grew (h). Panel (e) is shown at $2 \times$ magnification compared to panels ( $f-h)$.
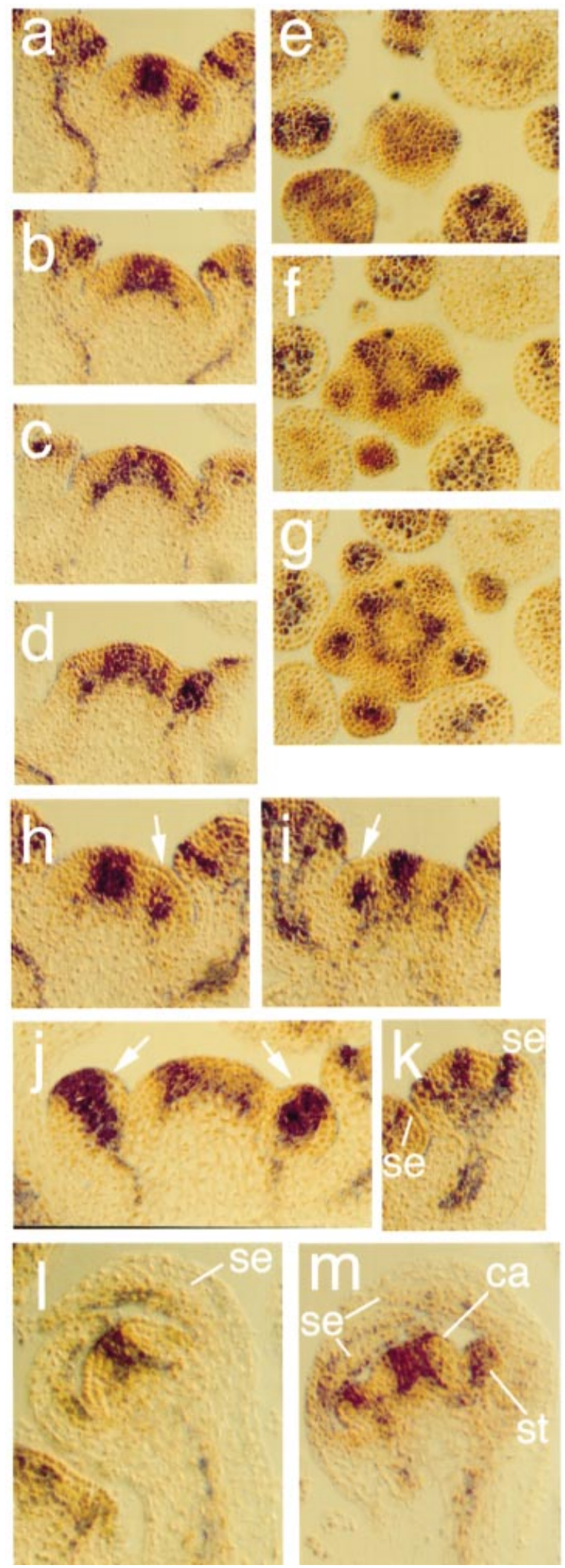


\section{REV encodes a homeodomain-containing protein}

To further analyse REV function, we isolated the $R E V$ gene. We localized REV to BAC MUP24 using a fine structure recombination mapping strategy with F2 progeny of rev-1 (No-0 ecotype) crossed to Ler (data not shown). One ORF (MUP24.4) failed to produce a PCR product in rev-8 due to a T-DNA insertion in intron 1. To verify that MUP24.4 corresponded to the REV gene, we determined the DNA sequence of this gene in wild type and three additional rev alleles. As summarized in Table 1 and Figure 6, we observed a lesion in each rev allele, indicating that ORF MUP24.4 corresponds to the $R E V$ gene.

The REV gene corresponds to the recently cloned INTERFASICULAR FIBERLESS1 (IFL1) gene, which is required for normal vascular tissue differentiation in stems (Ratcliffe etal., 2000; Zhong etal., 1997; Zhong and $\mathrm{Ye}, 1999)$. The REV/IFL1 gene contains 18 exons and encodes a predicted protein of 842 amino acids (Figure 6). The predicted REV protein contains both a homeodomain and a leucine zipper. REV is a member of the Arabidopsis HD-ZIP III subfamliy of HD-ZIP genes that includes ATHB8, ATHB9 and ATHB14 (Sessa etal., 1998). These genes all share a sterol-lipid binding domain, known as a START domain (Ponting and Aravind, 1999). The role of this domain in a putative transcription factor in unclear.

\section{REV expression predicts sites of lateral meristem formation}

If $R E V$ is required for the initiation of meristem activity at LSMs and FMs, its expression should precede the establishment of meristems in leaf axils and floral primordia. Specifically, its expression should precede the expression of the meristem regulators WUS, STM, CLV1 and CLV3. In addition, the pleiotropic phenotypic defects of rev/ifl1 mutants imply that $R E V$ is also expressed during organ development and in vascular tissue. To test these predictions, we carried out RNA in

Figure 8. $R E V$ is expressed in a complex manner in the shoot and flower meristem.

RNA in situ hybridizations with a REV antisense probe were performed on wild-type shoot apical meristems section longitudinally (a-d) and transversely $(\mathrm{e}-\mathrm{g})$. See text for discussion of expression pattern. In early stage 1 flowers, REV was expressed in a central group of L3 cells ( $h, i$, arrows). By the stage $1 /$ stage 2 boundary (j, right arrow), REV expression expanded to include L1 and L2 cells and continued in this pattern through stage 2 (j, left arrow). At stage 3, REV expression continued in the center of the meristem, but was also expressed on the adaxial face of sepal primordia (se) as well as internal vascular precursor cells (k). Central meristem expression continued through stage 5 (I), eventually comprising the adaxial face of carpel (ca) primordia at stage $6(\mathrm{~m})$. st, stamen primordium. situ hybridization experiments with embryo, leaf and floral tissue.

During embryo development, REV expression was detected as early as the heart stage (earlier stages were not tested). REV expression encompassed the adaxial portion of the cotyledon primordia, the SAM and the vascular precursor cells of the hypocotyl and root (Figure 7a-d). This expression pattern was maintained through at least the 'walking stick' stage, although at comparably lower levels.

Because LSM formation is delayed until the transition to flowering (Grbic and Bleecker, 2000), we examined REV expression within developing cauline leaves (Figure 7e-h). In addition to expression on the adaxial portion of the leaf and within the developing leaf vasculature, REV expression was detected at the earliest signs of LSM formation. At this point, REV was expressed in a small number of subepidermal cells. REV expression continued as the LSM was initiated, eventually taking on the expression pattern seen within the SAM (see below). Thus, REV was expressed at the earliest stages and throughout LSM initiation and development.

Within the SAM, REV exhibited a very complex expression pattern (Figure 8). When inflorescence SAMs were sectioned longitudinally and used for in situ hybridization, $R E V$ was detected in the center of the SAM in an invertedcup-shaped population of cells (Figure $8 a-d$ ). The cells in the very center of the meristem in the lower L3 cells did not express REV. These should correspond approximately to the WUS-expressing cells (Mayer et al., 1998). Above these cells, REV was expressed in L2 and the topmost L3 cells, but on the flanks of the meristem $R E V$ was expressed only in $L 3$ cells. SAMs sectioned transversely were also used for in situ hybridization (Figure $8 \mathrm{e}-\mathrm{g}$ ). These again revealed $R E V$ expression in $L 2$ and topmost $L 3$ cells in the center of the SAM. Interestingly, REV expression on the flanks of the meristem predicted sites of flower primordia formation. Using the existing organ primordia to establish the phyllotactic pattern, REV expression appeared to predict the next 3-5 sites of flower primordia initiation, making $R E V$ the earliest marker to date for organ anlage (Figure 9). No previous gene expression studies have identified primordia this early in development; however, the position of gene expression matches the predicted position of the incipient organs. Most significantly, these data show that $R E V$ was expressed prior to WUS.

In developing flowers, REV expression was detected prior to stage 1 in the L3 layer. In early stage 1 flowers, $R E V$ expression was detected in the center of the L3 layer, which is a pattern similar to that of WUS (Figure $8 \mathrm{~h}, \mathrm{i})$. During late stage 1, REV expression expanded to the central region of the $L 2$ and $L 1$ layers (Figure 8j). By stage 2, REV expression was at its highest and found in a pattern very similar to that for STM, namely, in all cell 
layers but excluded from presumptive sepal anlage (Long etal., 1996; Figure 8j). By stage 3, REV expression became more complex, with expression in the center of the flower meristem retained, but additional expression on the adaxial side of sepal primordia, as well as internal tissue connecting the base of the sepal primordia to the center of the flower stem (Figure 8k). $R E V$ expression was maintained in the center of the flower meristem through stages 4 and 5 (Figure 8l). By stage 6, carpel primordia are initiated in the center of the flower meristem (Smyth etal., 1990), and REVexpressing cells in the center constituted the adaxial side of the carpel primordia (Figure $8 \mathrm{~m}$ ). REV continued to be expressed on the adaxial carpel face, eventually being expressed in the placenta and ovules (data not shown). Within other organs, REV expression was largely on the adaxial portion of the primordia (data not shown).

$R E V$ is a member of a closely related gene family, and the family members most closely related to $R E V$ are $A T H B 9$, and $A T H B 14$. This raised the possibility that the $R E V$ expression pattern might, in part, represent cross hybridization to the ATHB9 and ATHB14 genes. Using in vitro translated RNAs and hybridization and wash conditions identical to those used in in situ hybridzation experiments, we found that the REV antisense probe hybridized at least 1000 times more strongly with the REV RNA than ATHB14 or ATHB9 RNA (see Supplementary Material at end of paper, Figure S1). Thus, under the conditions used for in situ hybridization, the $R E V$ probe specifically detects REV mRNA.

\section{Discussion}

We have investigated the phenotype and genetic interactions of mutations in the REV gene, focusing on the role of $R E V$ in lateral meristem formation. Our observations indicate that $R E V$ is required for lateral meristem initiation, and that $R E V$ likely acts in the same pathway as, and upstream of several known meristem regulators. $R E V$ encodes a predicted transcription factor whose expression is consistent with an early role in meristem initiation.

\section{$\mathrm{REV}$ is required for lateral meristem activity}

Several lines of evidence indicate that $R E V$ is required to establish meristems in leaf axils and floral primordia. First, phenotypic analysis indicates that many leaves and flowers in rev mutants lack meristem activity. In leaf axils this is characterized by a complete absence of growth in the lateral position. Floral primordia lacking meristem activity develop into flowers containing sepals and petals but lacking stamens and carpels, and this is the floral phenotype most commonly observed in rev mutants. While it may be unclear how a flower lacking meristem activity would be able to develop multiple organs, the wus mutant, which appears to lack meristem activity within the flower as well, develops a similar pattern of flower organs (Laux etal., 1996). Floral primordia in rev mutants also occasionally develop into filamentous structures, which may represent a more severe loss of cell division activity.

Second, although clv mutations dominantly restore meristem formation and activity within the severely affected stm-1 mutant plants, they have no effect on the development of LSMs or the number of organs initiated by reduced flowers in a rev mutant background. This suggests that rev mutants lack meristem activity, and hence CLV function.

Third, rev does not display additive interactions with the partial-loss-of-function stm-2 allele. The flowers of rev stm-2 plants develop a similar complement of organs as either single mutant, suggesting that REV and STM act in a common pathway. This is consistent with a role for $R E V$ in activating STM activity, and is supported by gene expression studies (see below).

Finally, additional genetic studies indicate that $R E V$ is required for ectopic and adventitious meristem formation in wus, stm, ap1 and ap1 cal mutant plants. Thus, all postembryonic shoot and flower meristems, and all ectopic meristems of this sort (i.e. non-root meristems) require $R E V$ activity.

\section{Rev expressivity and ecotype modifiers}

Despite a fairly clear role for REV in LSM and FM activity, many LSMs and FMs develop normally within rev mutants. This implies the existence of redundant factors that are capable of carrying out lateral meristem initiation in the absence of REV activity. One possibility is that the very closely related ATHB9 and ATHB14 genes are capable of acting redundantly with REV. A lower or different expression pattern for these two genes could account for the rev phenotype and expressivity. An interesting corollary hypothesis would be that the modifiers of the $\mathrm{Rev}^{-}$phenotype in the various ecotypes could represent variability in ATHB9 and $A T H B 14$ expression.

\section{REV has multiple functions}

$R E V$ functions can be separated into three categories: (1) $R E V$ is required for lateral meristem activity, as described above; (2) REV is required for normal organ development, as described in detail previously (Talbert et al., 1995); (3) REV is required for proper differentiation of vascular-associated elements within the stem, as described for if1l mutants (Zhong etal., 1997). The data 
Figure 9. REV expression marks incipient flower primordia.

RNA in situ hybridizations with a REV antisense probe were performed on transversely sectioned inflorescence shoot apical meristems. For each of three different meristems, expression is shown on the left $(\mathrm{a}, \mathrm{c}, \mathrm{e})$. On the right, flower primordia are numbered from the youngest, and the predicted pattern of incipient flower primordia based on the phyllotaxy of existing flowers is indicated with a blue line (b,d,f). Note that $R E V$ was expressed in patches of cells in the next 3-5 primordia.
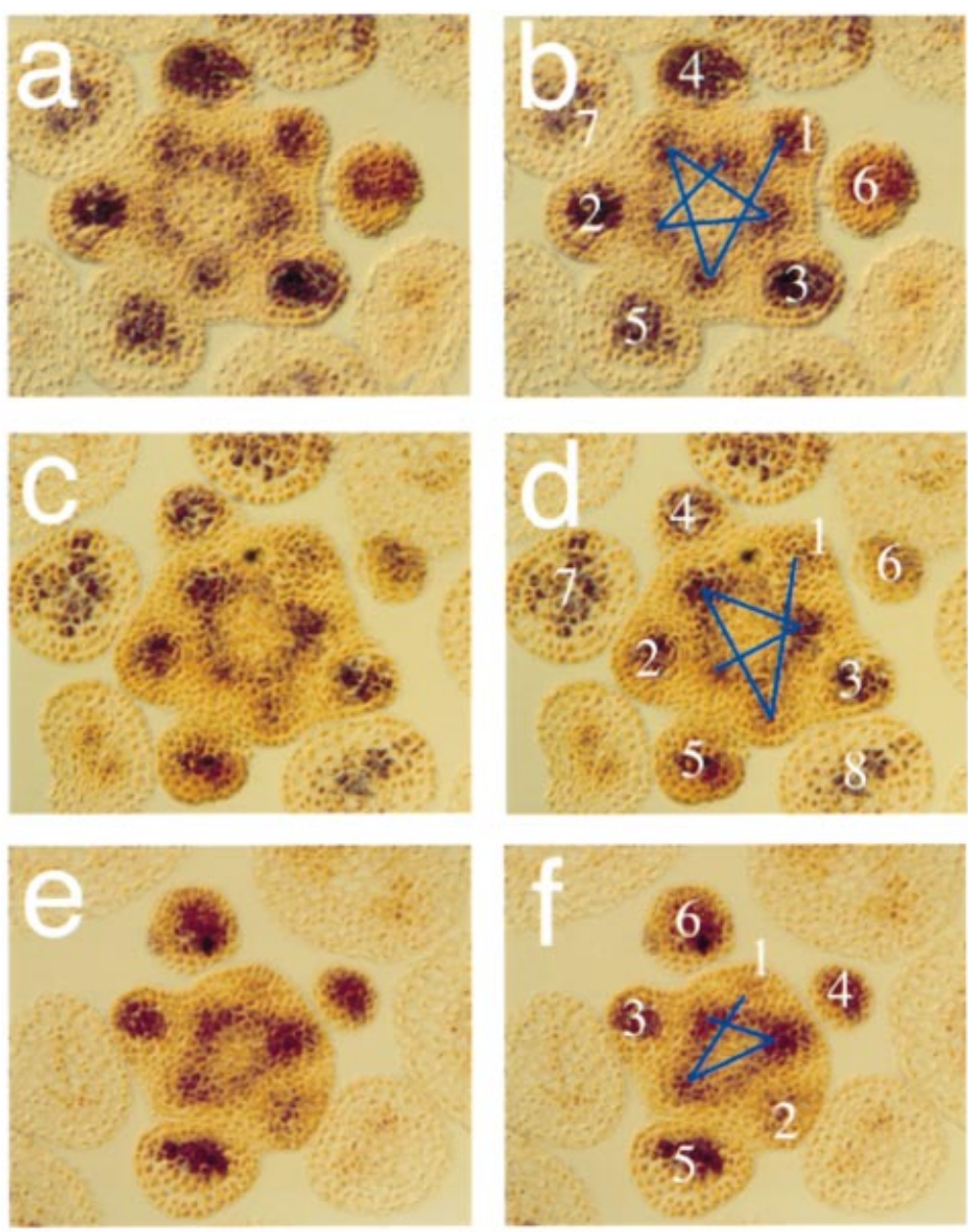

we present here suggest that these activities can be functionally separated. Rev mutant alleles in the Ler ecotype exhibit lateral meristem defects without any apparent organs defects. This suggests that the lateral meristem defects are not an indirect result of organ defects. In addition, there is no absolute correlation between organ defects and lateral meristem defects when segregating populations of rev in Ler crossed into Columbia were examined. In other words, the plants with the most severely affected organs did not always have the most severe reduction in lateral meristem activity. While there was a general correlation, we suggest this is due to common redundant elements affecting both organ and meristem phenotypes. Finally, expression patterns are consistent with each of these activities constituting a separate function (see below).

\section{REV expression matches predicted function}

Each of the hypotheses of REV function based on genetic and phenotypic analysis makes specific predictions about the expression pattern of the $R E V$ gene. If $R E V$ is required for LSM and FM activity, REV should be expressed at the earliest stages of lateral meristem development. If $R E V$ has multiple separate functions within lateral meristems, organs and vasculature, then REV should be expressed separately within each of these tissues.

Within the SAM, the complex REV expression pattern matches the function predicted by phenotypes. $R E V$ is the earliest marker to date for flower primordia. Based on phyllotaxy of existing organs, REV appears to mark the next three to five flower primordia forming on the flanks of the shoot meristem. Thus REV expression precedes that of all other FM regulators. Curiously, REV expression appears in the same position within stage 1 flowers as the earliest known regulator of FM activity, WUS. WUS is expressed in a central group of L3 cells within the stage 1 FM (Mayer et al., 1998), and REV is found in a similar pattern. The next meristem regulator expressed is STM at stage 2, which is found throughout the cell layers everywhere except the sepal anlage (Long et al., 1996). REV is found in a coincident pattern by stage 2 . We propose that REV acts either indirectly to establish meristem identity, or directly to

(C) Blackwell Science Ltd, The Plant Journal, (2001), 25, 223-236 
activate the expression of other meristem regulators. The observations that REV expression matches that of WUS and $S T M$ at their initiation and that REV encodes a putative transcription factor may make direct activation a plausible hypothesis.

Within developing LSMs, REV appears to be expressed coincident with the earliest morphological establishment of an axillary meristem. $R E V$ and $S T M$ (Long and Barton, 2000) appear to be the earliest markers for these developing meristems. We hypothesize that within these cells $R E V$ acts either indirectly to establish meristem identity, or directly to activate the expression of other meristem regulators.

Within organs, REV is expressed largely on the adaxial portion of the organ. A number of genes are expressed specifically on one 'face' (i.e. abaxial or adaxial) of leaf and flower organs, including CRABS CLAW (Bowman and Smyth, 1999), INNER NO OUTER (Villanueva et al., 1999), FIL, YABBIE2 (YAB2), YAB3 (Siegfried et al., 1999), ZWILLE/ PINHEAD (Lynn etal., 1999; Moussian etal., 1998), and PROLIFERA (Springer et al., 2000). Many of these genes have been shown to be required for abaxial or adaxial fate. By analogy, REV may be involved in establishing adaxial fate in cotyledons, leaves and floral organs. In this regard, the loss of axillary meristems in rev mutants may be an indirect consequence of loss of adaxial cell fate in leaves, which has been proposed to promote axillary meristem formation (McConnell and Barton, 1998; Siegfried etal., 1999).

$R E V$ is also expressed in developing vasculature. Shortly after the initiation of sepal primordia within the flower, REV appears to mark vascular precursor cells that will link the sepals to the main vascular cells in the center of the flower pedicel. Additional expression of REV is observed within vascular precursors within the inflorescence stem and developing flower organs. A detailed analysis of rev/if1l mutants has already established the role of REV within the developing stem (Zhong et al., 1997).

A critical questioned raised is how REV can act in three different tissue types to carry out distinct developmental programs. Perhaps REV regulates a single common process (e.g. cell division) in each tissue. Alternatively, $R E V$ may interact with different transcription factors in each tissue.

\section{Experimental procedures}

\section{Plant growth and tissue processing}

The isolation of the rev-6 (vam-1), rev-7 (vam-2), and rev-8 (tj-72) were previously described (Chen et al., 1999; Pogany et al., 1998). All plants were in the Landsberg erecta background expect those specficially mentioned in the text.

Seeds were sown on a $1: 1: 1 \mathrm{mix}$ of top soil: perlite: vermiculite and imbibed for 7 days at $4^{\circ} \mathrm{C}$. Plants were grown at $22^{\circ} \mathrm{C}$ under approximately 800 -foot-candles of constant cool white fluorescent light. Plants were fertilized once a week.
Tissue and image processing for scanning electron microscopy (SEM) was carried out as described previously (Yu etal., 2000).

Post-embryonic organ formation for the genotypes indicated in Figure 4 was performed as follows. The description is for stm-1, and is applicable to all genotypes analysed. Progeny of a heterozygote $\mathrm{stm}-1 /+$ plant were sterilized and germinated in $0.5 \times$ MS media supplemented with $1 \%$ sucrose. After 7 days' treatment at $4^{\circ} \mathrm{C}$, plates were moved to $20^{\circ} \mathrm{C}$ at $\sim 150$-foot-candles of cool white fluorescent light. At regular intervals, plates were opened under sterile conditions and examined with a stereo microscope. All plants not homozygous for $s t m-1$ were identified within 8-10 days and removed. Remaining plants were monitored for any postembryonic organ formation. Plants that did not survive the entire 35-day period were not included in any calcualtions.

\section{RNA in situ hybridization}

Nonradioactive in situ hybridization experiments were carried out as previously described (Klucher etal., 1996) except that tissue was fixed and embedded as described by (Vielle-Calzada et al., 1999). REV antisense probes were made from cDNA clone Col\#19. The probe contained nucleotides 680 (relative to the ATG; Eae I recognition sequence) to the end of the mRNA.

\section{Acknowledgements}

We thank Ephraim Simon and Tom Jack for isolating the rev-6 and rev-7 alleles, respectively, and David Bay for photographic assistance. The work was supported by grants from the National Science Foundation (NSF) Developmental Mechanisms program (IBN-9816760) to S. E. C. We thank the DNA Sequencing Facility at the University of Utah, supported in part by $\mathrm{NCl}$ grant \#5p30CA42014. The scanning electron microscope used was acquired under grant BSR-83-14092 from the NSF.

\section{References}

Aida, M., Ishida, T., Fukaki, H., Fujisawa, H. and Tasaka, M. (1997) Genes involved in organ separation in Arabidopsis - an analysis of the cup-shaped cotyledon mutant. Plant Cell, 9, 841-857.

Alvarez, J. (1994) The SPITZEN gene. In Arabidopsis: An Atlas of Morphology and Development (Bowman, J.L., ed. ). New York: Springer-Verlag, pp. 188-189.

Barton, M.K. and Poethig, R.S. (1993) Formation of the shoot apical meristem in Arabidopsis thaliana: an analysis of development in the wild type and shoot meristemless mutant. Development, 119, 823-831.

Bohmert, K., Camus, I., Bellini, C., Bouchez, D., Caboche, M. and Benning, C. (1997) AGO1 defines a novel locus of Arabidopsis controlling leaf development. EMBO J. 17, 170-180.

Bowman, J.L., Alvarez, J., Weigel, D., Meyerowitz, E.M. and Smyth, D.R. (1993) Control of flower development in Arabidopsis thaliana by APETALA1 and interacting genes. Development, 119, 721-743.

Bowman, J.L. and Smyth, D.R. (1999) CRABS CLAW, a gene that regualtes carpel and nectary development in Arabidopsis, encodes a novel protein with zinc fingers and helix-loop-helix domains. Development, 126, 2387-2396.

Chen, Q., Atkinson, A., Ostuga, D., Christensen, T., Reynolds, L. and Drews, G.N. (1999) The Arabidopsis FILAMENTOUS 
FLOWER gene is required for flower formation. Development, 126, 2715-2726.

Clark, S.E. (1997) Organ formation at the vegetative shoot meristem. Plant Cell, 9, 1067-1076.

Clark, S.E., Jacobsen, S.E., Levin, J. and Meyerowitz, E.M. (1996) The CLAVATA and SHOOT MERISTEMLESS loci competitively regulate meristem activity in Arabidopsis. Development, 122, 1567-1575.

Clark, S.E., Running, M.P. and Meyerowitz, E.M. (1993) CLAVATA1, a regulator of meristem and flower development in Arabidopsis. Development, 119, 397-418.

Clark, S.E., Running, M.P. and Meyerowitz, E.M. (1995) CLAVATA3 is a specific regulator of shoot and floral meristem development affecting the same processes as CLAVATA1. Development, 121, 2057-2067.

Clark, S.E., Williams, R.W. and Meyerowitz, E.M. (1997) The CLAVATA1 gene encodes a putative receptor kinase that controls shoot and floral meristem size in Arabidopsis. Cell, 89, 575-585.

Endrizzi, K., Moussain, B., Haecker, A., Levin, J.Z. and Laux, T. (1996) The SHOOT MERISTEMLESS gene is required for maintenance of undifferentiated cells in Arabidopsis shoot and floral meristems and acts at a different regulatory level than the meristem genes WUSCHEL and ZWILLE. Plant J. 10, 967-979.

Eshed, Y. and Bowman, J.L. (2000) Formation and maintenance of the shoot apical meristem. Trends Plant Sci. 5, 110-115.

Fletcher, J.C., Brand, U., Running, M.P., Simon, R. and Meyerowitz, E.M. (1999) Signaling of cell fate decisions by CLAVATA3 in Arabidopsis shoot meristems. Science, 283, 1911-1914.

Grbic, V. and Bleecker, A.B. (2000) Axillary meristem development in Arabidopsis. Plant J. 21, 215-223.

Jacobsen, S.E., Running, M.P. and Meyerowitz, E.M. (1999) Disruption of an RNA helicase/RNAse III gene in Arabidopsis causes unregulated cell division in floral meristems. Development, 126, 5231-5243.

Jürgens, G., Ruiz, R.A.T., Laux, T., Mayer, U. and Berleth, T. (1994) Early events in apical-basal pattern formation in Arabidopsis. In Plant Molecular Biology: Molecular-Genetic Analysis of Plant Development and Metabolism (Coruzzi, G. and Puigdomenech, P., eds). Berlin: Springer, pp. 95-103.

Kayes, J.M. and Clark, S.E. (1998) CLAVATA2, a regulator of meristem and organ development in Arabidopsis. Development, 125, 3843-3851.

Klucher, K.M., Chow, H., Reiser, L. and Fischer, R.L. (1996) The AINTEGUMENTA gene of Arabidopsis required for ovule and female gametophyte development is related to the floral homeotic gene APETALA2. Plant Cell, 8, 137-153.

Laufs, P., Grandjean, O., Jonak, C., Kiêu, K. and Traas, J. (1998) Cellular parameters of the shoot apical meristem in Arabidopsis. Plant Cell, 10, 1375-1389.

Laux, T., Mayer, K.F.X., Berger, J. and Jurgens, G. (1996) The WUSCHEL gene is required for shoot and floral meristem integrity in Arabidopsis. Development, 122, 87-96.

Long, J.A. and Barton, M.K. (1998) The development of apical embryonic pattern in Arabidopsis. Development, 125, 3027-3035.

Long, J. and Barton, M.K. (2000) Initiation of axillary and floral meristems in Arabidopis. Dev. Biol. 218, 341-353.

Long, J.A., Moan, E.I., Medford, J.I. and Barton, M.K. (1996) A member of the KNOTTED class of homeodomain proteins encoded by the STM gene of Arabidopsis. Nature, 379, $66-69$.
Lynn, K., Fernandez, A., Aida, M., Sedbrook, J., Tasaka, M., Masson, P. and Barton, M.K. (1999) The PINHEAD/ZWILLE gene acts pleiotropically in Arabidopsis development and has overlapping functions with the ARGONAUTE1 gene. Development, 126, 469-481.

Mayer, K.L., Schoof, H., Haecker, A., Lenhard, M., Jürgens, G. and Laux, T. (1998) Role of WUSCHEL in regulating stem cell fate in the Arabidopsis shoot meristem. Cell, 95, 805-815.

McConnell, J.R. and Barton, M.K. (1998) Leaf polarity and meristem formation in Arabidopsis. Development, 125, 2935-2942.

Moussian, B., Schoof, H., Haecker, A., Jürgens, G. and Laux, T. (1998) Role of the ZWILLE gene in the regulation of central shoot meristem cell fate during Arabidopsis embryogenesis. EMBO J. 17, 1799-1809.

Pogany, J.A., Simon, E.J., Katzman, R.B., de Guzman, B.M., Yu, L.P., Trotochaud, A.E. and Clark, S.E. (1998) Identifying novel regulators of shoot meristem development. J. Plant Res. 111, 307-313.

Ponting, C.P. and Aravind, L. (1999) START: a lipid-binding domain in StAR, HD-ZIP and signalling proteins. Trends Biochem. Sci. 24, 130-132.

Ratcliffe, O.J., Riechmann, J.L. and Zhang, J.Z. (2000) INTERFASCICULAR FIBERLESS1 is the same gene as REVOLUTA. Plant Cell, 12, 315-317.

Schumacher, K., Schmitt, T., Rossberg, M., Schmitz, G. and Theres, K. (1999) The Lateral suppressor ( $L s)$ gene of tomato encodes a new member of the VHIID protein family. Proc. Natl Acad. Sci. USA, 96, 290-295.

Sessa, G., Steindler, C., Morelli, G. and Ruberti, I. (1998) The Arabidopsis Athb-8-9 and -14 genes are members of a small gene family coding for highly related HD-ZIP proteins. Plant Mol. Biol. 38, 609-622.

Siegfried, K.R., Eshed, Y., Baum, S.F., Otsuga, D., Drews, G.N. and Bowman, J.L. (1999) Members of the YABBY gene family specify abaxial cell fate in Arabidopsis. Development, 126, 4117-4128.

Smyth, D.R., Bowman, J.L. and Meyerowitz, E.M. (1990) Early flower development in Arabidopsis. Plant Cell, 2, 755-767.

Springer, P.S., Holding, D.R., Groover, A., Yordan, C. and Martienssen, R.A. (2000) The essential $\mathrm{Mcm} 7$ protein PROLIFERA is localized to the nucleus of dividing cells during the G (1) phase and is required maternally for early Arabidopsis development. Development, 127, 1815-1822.

Steeves, T.A. and Sussex, I.M. (1989) Patterns in Plant Development. New York: Cambridge University Press.

Stirnberg, P., Chatfield, S.P. and Leyser, H.M.O. (1999) AXR1 actis after lateral bud formation to inhibit late growth in Arabidopsis. Plant Physiol. 121, 839-847.

Talbert, P.B., Alder, H.T., Parks, D.W. and Comai, L. (1995) The REVOLUTA gene is necessary for apical meristem development and for limiting cell divisions in the leaves and stems of Arabidopsis thaliana. Development, 121, 2723-2735.

Vielle-Calzada, J.-P., Thomas, J., Spillane, C., Coluccio, A., Hoeppner, M.A. and Grossniklaus, U. (1999) Maintenance of genomic imprinting at the Arabidopsis medea locus requires zygotic DDM1 activity. Genes Dev. 13, 2971-2982.

Villanueva, J.M., Broadhvest, J., Hauser, B.A., Meister, R.J., Schneitz, K. and Gasser, C.S. (1999) INNER NO OUTER regulates abaxial-adaxial patterning in Arabidopsis ovules. Genes Dev, 13, 3160-3169.

Yu, L.P., Simon, E.J., Trotochaud, A.E. and Clark, S.E. (2000) POLTERGEIST functions to regulate meristem development 
236 Denichiro Otsuga et al.

downstream of the CLAVATA loci. Development, 127, 1661-1670.

Zhong, R., Taylor, J.J. and Ye, Z.H. (1997) Disruption of interfascicular fiber differentiation in an Arabidopsis mutant. Plant Cell, 9, 2159-2170.
Zhong, R. and Ye, Z.H. (1999) IFL1, a gene regulating interfascicular fiber differentiation in Arabidopsis, encodes a homeodomain-leucine zipper protein. Plant Cell, 11, 2139-2152. 